\title{
Structural and Magnetic Properties of Cobalt Ferrite Nanopowders Synthesis Using Contact Non-Equilibrium Plasma
}

\author{
L. Frolova*, A. Derimova and T. Butyrina \\ Ukrainian State Chemical Technology University, 49005, Gagarin, 8, Dnipro, Ukraine
}

\begin{abstract}
Different grades of magnetic cobalt ferrite $\left(\mathrm{Co}_{x} \mathrm{Fe}_{3-x} \mathrm{O}_{4}\right)$ nanoparticles were synthesized with various molar ratios of $\mathrm{Fe}^{2+}$ to $\mathrm{Co}^{2+}$ ions in the initial salt solutions method with using contact non-equilibrium plasma. The crystal structure and morphology of the nanoparticles are obtained from X-ray diffraction and transmission electron microscopy studies. With an increase in the value of $x$, the saturation magnetization for the samples of $\mathrm{CoFe}_{2} \mathrm{O}_{4}$ has decreased from $150.9 \mathrm{emu} / \mathrm{g}$ to $1.2 \mathrm{emu} / \mathrm{g}$. The dependence of the coercive force on $x$ is extremal with a plateau maximum corresponding to the $x=0.5-1.25$.
\end{abstract}

DOI: 10.12693/APhysPolA.133.1021

PACS/topics: nanomaterials, cobalt, ferrite, Fe/Co molar ratio

\section{Introduction}

The interest in ferrite nanoparticles has grown significantly over the past decade. This is due to their use in radio engineering, microwave technology, HD-technologies, in computing and modeling devices, proximity switches, and amplifiers. The highly disperse ferrite of cobalt is one of the starting materials for the production of magnetic powders used as a component in audio and video recording media. One way to improve the magnetic properties of ferrite powders is to improve the starting material structure [1-3].

The analysis of the latest publications and patents on the synthesis of magnetic dispersive nanoparticles or magnetic fluids (MF) have shown that attempts to find cheaper methods of production are still active, taking into account the ever increasing volume of the consumption of such materials [4-6].

\section{Experimental part}

Aqueous solutions of cobalt sulfate hexahydrate and ferrous sulfate hexahydrate have been used as starting precursors. The preparation of nanosized cobalt ferrite has been carried out on a laboratory plasma chemical plant, which consists of a single-stage plasma reactor of a discrete type, a step-up transformer, a transformerigniter and a vacuum pump. The $\mathrm{pH}$ of the solution was monitored at regular intervals, and the product obtained was washed and dried for further investigation. Infrared (IR) reflection spectra of cobalt ferrites $\mathrm{Co}_{x} \mathrm{Fe}_{3-x} \mathrm{O}_{4}$ $(x=0.25,0.5,0.75,1.0,1.5,2.0)$ were measured within a $400-4000 \mathrm{~cm}^{-1}$ range by employing a Fourier transform infrared (FTIR) spectrometer Nicolet iS10. The

*corresponding author; e-mail: 19kozak83@gmail.com phase composition and structure of ferrite samples was studied using X-ray diffractometer DRON-2 with $\mathrm{Cu} K_{\alpha}$ radiation. Transmission electron microscope PEM $125 \mathrm{~K}$ (Selmi, Ukraine) was used for characterization of particle's size and morphology of the obtained samples. The magnetic properties of the final powder have been studied using a vibrating sample magnetometer.

TABLE I

Code and composition of the $\mathrm{Co}_{x} \mathrm{Fe}_{3-x} \mathrm{O}_{4}$ samples obtained

\begin{tabular}{c|c|c|c|c|c|c}
\hline \hline Code of the sample & A1 & A2 & A3 & A4 & A5 & A6 \\
\hline $\begin{array}{c}x \text { in the formula } \\
\mathrm{Co}_{x} \mathrm{Fe}_{3-x} \mathrm{O}_{4}\end{array}$ & 2 & 1.5 & 1.0 & 0.75 & 0.5 & 0.25
\end{tabular}

\section{Results and discussion}

X-ray patterns of samples A1-A6 with different values of $x$ look similar (Fig. 1), corresponding to spinel oxide structure of cobalt ferrite. But the samples A6, A5 contain additional phases. Samples A4-A6 are crystalline. All main characteristic peaks of spinels (220), (311), (222), (400), (422), (511) are present on the X-ray diffraction patterns. Moreover, with the cobalt content increase, the diffraction peaks have broadened, and their intensity has decreased due to the formation of the amorphous $\mathrm{Co}_{3} \mathrm{O}_{4}$. It is established in $[7-9]$ that under different synthesis conditions and values $x$, both the formation of $\gamma-\mathrm{Fe}_{2} \mathrm{O}_{3} \cdot \mathrm{H}_{2} \mathrm{O}$ structure, and the spinel structure of the type $\mathrm{Co}_{x} \mathrm{Fe}_{3-x} \mathrm{O}_{4}$ have been possible. In this case, the process is likely to proceed according to the second scheme for samples A3-A5, which can be confirmed by IR spectroscopy and microscopy data. Samples A4-A6 contain a Fe-rich composition which leads to the formation of a spinel structure such as magnetite, hausmanite and lepidocrocite. In addition, in the case of sample A2, a Co-rich composition probably crystallizes in the structure type of $\mathrm{Co}_{3} \mathrm{O}_{4}$. 
The relative redox potential of metal ion between bivalent and trivalent states in suspension medium has been known to decrease in order of $\mathrm{Co}>\mathrm{Fe}$. This probably causes the predominant formation of an iron hydroxocomplex.

For example, based on the standard reduction potentials for the metals:

$$
\begin{aligned}
& \mathrm{Fe}^{3+}+1 \mathrm{e}^{-} \rightarrow \mathrm{Fe}^{2+}, E_{0}=0.77 \mathrm{~V}, \\
& \mathrm{Co}^{3+}+1 \mathrm{e}^{-} \rightarrow \mathrm{Co}^{2}+, E_{0}=1.81 \mathrm{~V} .
\end{aligned}
$$

It can be considered that the reduction of $\mathrm{Co}^{3+}$ by $\mathrm{Fe}^{2+}$ is thermodynamically favorable as shown by the following equations:

$$
\mathrm{Fe}^{2+}+\mathrm{Co}^{3+} \rightarrow \mathrm{Fe}^{3+}+\mathrm{Co}^{2+}, E_{0}=1.04 \mathrm{~V} .
$$

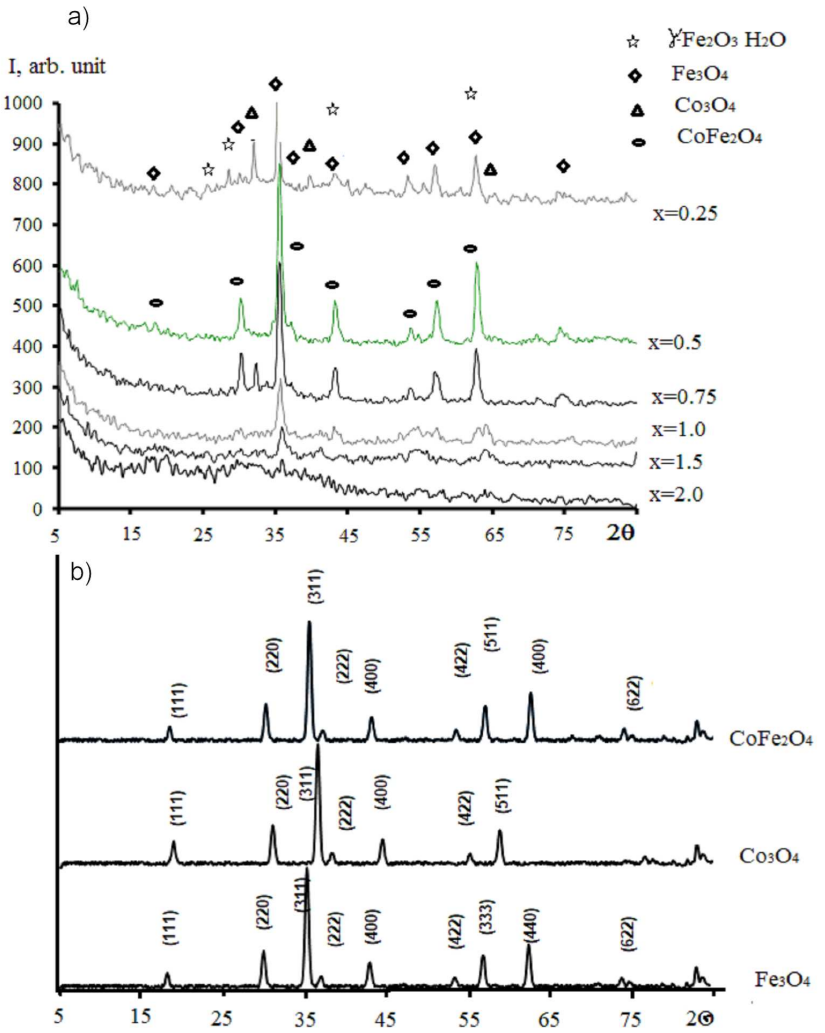

Fig. 1. X-ray pattern of the samples (a) numbering corresponds to Table I, (b) the samples pure $\mathrm{CoFe}_{2} \mathrm{O}_{4}$, $\mathrm{Co}_{3} \mathrm{O}_{4}, \mathrm{Fe}_{3} \mathrm{O}_{4}$.

Therefore, one can envisage that $\mathrm{Fe}^{2+}$ in the oxide could reduce the $\mathrm{Co}^{3+}$ surface species. This reduction could take place by an electron transfer process within the semiconductor oxide structure and leads to the formation of amorphous phases.

Figure 2 shows a photomicrograph of stoichiometric ferrite of composition A3.

$\mathrm{CoFe}_{2} \mathrm{O}_{4}$ nanoparticles have a spherical shape, their size varying from 10 to $100 \mathrm{~nm}$. Micrographs also show the faces that confirm the secondary growth of primary particles under the influence of CNP.

To test the existence of certain secondary phases, the initial samples of $\mathrm{Co}_{x} \mathrm{Fe}_{3-x} \mathrm{O}_{4}$ have also been studied

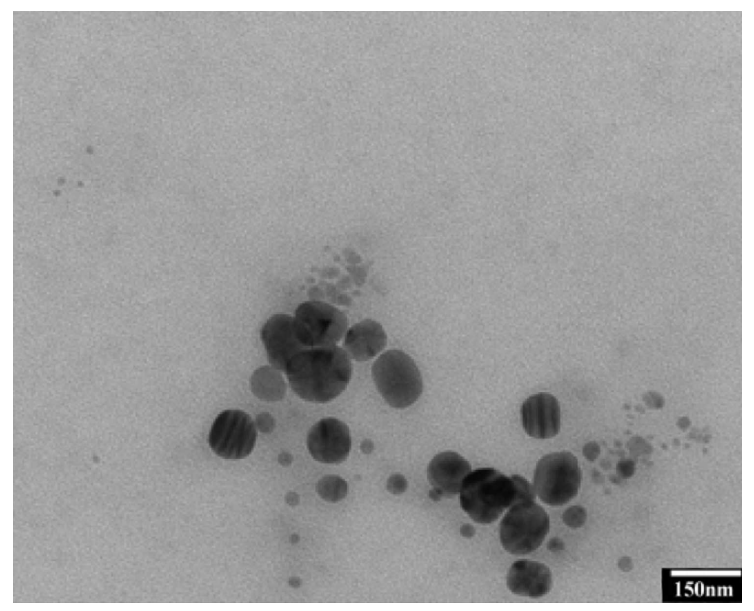

Fig. 2. TEM images of $\mathrm{CoFe}_{2} \mathrm{O}_{4}$ samples.

by FTIR spectrometry in the range of $400-4000 \mathrm{~cm}^{-1}$ (Fig. 3). The intense wide absorption band at $3450 \mathrm{~cm}^{-1}$ corresponds to the valence vibrations of metal hydroxyl $(\mathrm{Fe}-\mathrm{OH}$ and $\mathrm{Co}-\mathrm{OH})$.

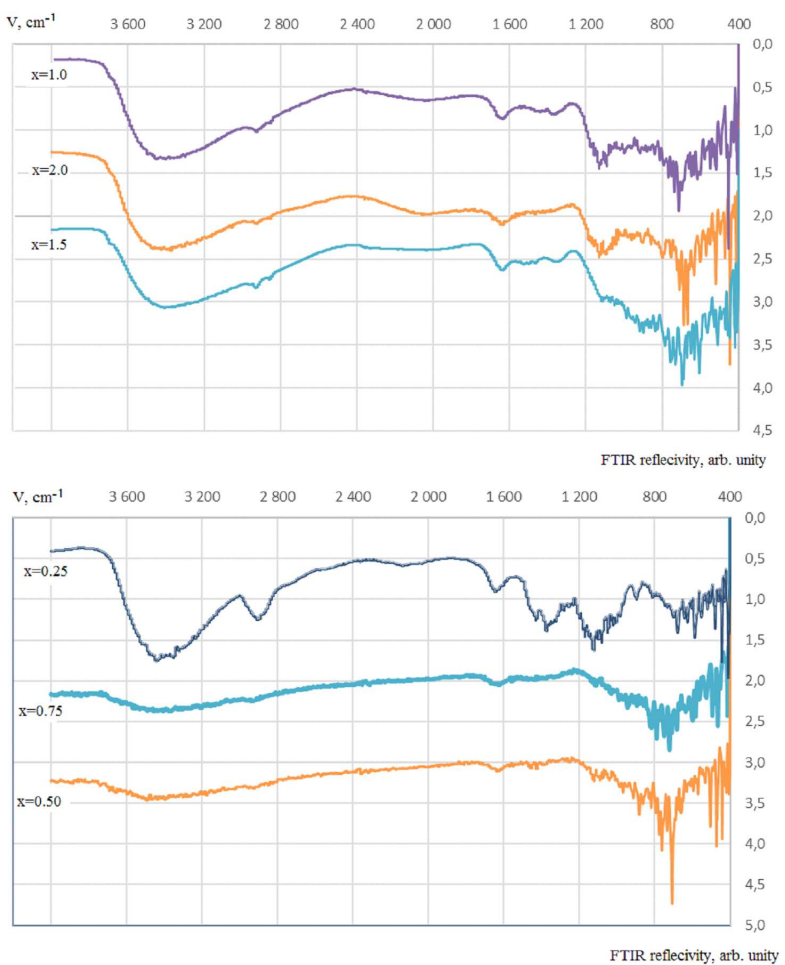

Fig. 3. Fourier spectra of the samples (Table I).

The wide branch at $3000-3150 \mathrm{~cm}^{-1}$ corresponds to the presence of water in the interlayer space (oscillations of the $\mathrm{O}-\mathrm{H}$ bond) for samples A1-A3. Moreover, the presence of the peak at $1630 \mathrm{~cm}^{-1}$ and its shoulder at $1500-1600 \mathrm{~cm}^{-1}$ confirms the presence of interlayer water (oscillations of the $\mathrm{H}-\mathrm{O}-\mathrm{H}$ bond). The presence of 
a large amount of bound water in samples A1-A3 correlates with X-ray pattern. The peaks of 1140, 1100, $980 \mathrm{~cm}^{-1}$ correspond to stretching vibrations of $\mathrm{Fe}-\mathrm{O}-\mathrm{H}$ and are manifested to a greater extent for samples A1A3. The standard line lepidocrocite phases cannot be distinguished from the $\mathrm{CoFe}_{2} \mathrm{O}_{4}$ phase from the XRD pattern. Therefore, the FTIR-spectrum is employed to check the presence of lepidocrocite in the $\mathrm{CoFe}_{2} \mathrm{O}_{4}$ nanoparticles. The peak at $1320 \mathrm{~cm}^{-1}$ corresponding to the ferrihydrite phase. The bands at $1320-1400 \mathrm{~cm}^{-1}$ is due to the magnetite-like modification of ferrihydrite. It has also been reported in the literature that the band at $1320 \mathrm{~cm}^{-1}$ correspond to lepidocrocite $\left(\gamma-\mathrm{Fe}_{2} \mathrm{O}_{3} \mathrm{H}_{2} \mathrm{O}\right)$ present in sample A6.

The two main broad metal-oxygen bands are known to be observed in IR spectra of ferrites: $v_{1}$ is usually observed in the range of $600-500 \mathrm{~cm}^{-1}$, corresponding to the intrinsic valence vibrations of the metal in the tetrahedral position of $\mathrm{M}_{\text {tetra }}-\mathrm{O}$ in $\mathrm{CoFe}_{2} \mathrm{O}_{4}$; and $v_{2}$ corresponds to $\mathrm{M}_{\text {octa }}-\mathrm{O}$ in the range of $400-450 \mathrm{~cm}^{-1}$ [10].

From these magnetization curves (Fig. 4) it follows that the synthesized samples have a similar magnetic behavior. The magnetic curves show a high coercive field due to the high magnetic anisotropy of cobalt ferrite. With an increase in the value of $x$, the saturation magnetization for the samples of $\mathrm{CoFe}_{2} \mathrm{O}_{4}$ has decreased from $140 \mathrm{emu} / \mathrm{g}$ to $1.2 \mathrm{emu} / \mathrm{g}$, respectively. The dependence of the coercive force on $x$ is extremal with a maximum corresponding to the stoichiometric ferrite, $\mathrm{CoFe}_{2} \mathrm{O}_{4}$. Comparison of magnetic properties of the as-synthesized cobalt ferrites and the reported $\mathrm{CoFe}_{2} \mathrm{O}_{4}$ measured at room temperature is shown in Table II.

TABLE II

The magnetic properties of cobalt ferrites synthesized by different methods.

\begin{tabular}{c|c|c|c|c}
\hline \hline Reference & $\begin{array}{c}\text { Particle } \\
\text { size } \\
{[\mathrm{nm}]}\end{array}$ & $\begin{array}{c}\mathrm{H}_{c} \\
{[\mathrm{Oe}]}\end{array}$ & $\begin{array}{c}\mathrm{M}_{s} \\
{[\mathrm{emu} / \mathrm{g}]}\end{array}$ & Method \\
\hline$[11]$ & 5.5 & 11 & 50 & hydrothermal \\
\hline$[12]$ & 11.7 & 286.0 & 58.4 & Co-precipitation \\
\hline$[12]$ & 5.58 & 23.7 & 12.6 & normal micelles \\
\hline$[12]$ & 7.63 & 25.2 & 29.4 & reverse micelles \\
\hline this work & 70 & 440 & 140 & $\begin{array}{c}\text { contact } \\
\text { non-equilibrium } \\
\text { plasma }\end{array}$
\end{tabular}

\section{Conclusion}

The reported comparative study of cobalt ferrites $\mathrm{Co}_{x} \mathrm{Fe}_{3-x} \mathrm{O}_{4}$ with different compositions $(x=0.25-2.0)$ has led us to the following conclusions:

1. Compositions other than stoichiometric compounds lead to the formation of additional phases depending on the composition, as evidenced by X-ray diffractometry and IR spectrometry.

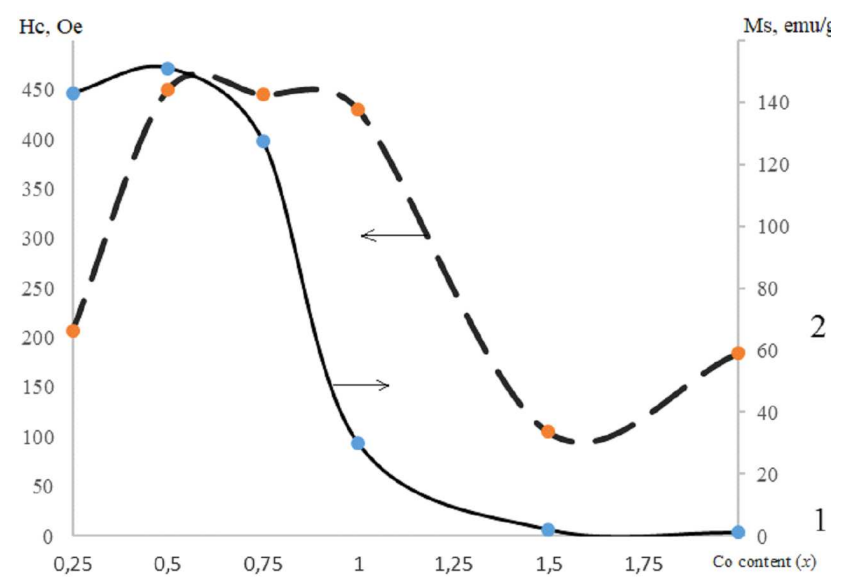

Fig. 4. Dependence of the coercive force and the saturation magnetization on $x: 1$ - the saturation magnetization, 2 - coercive force.

2. Pure cobalt ferrite $\mathrm{CoFe}_{2} \mathrm{O}_{4}$ with a crystallite size less than $100 \mathrm{~nm}$ has been synthesized by the coprecipitation method followed by CNP treatment with molar ratio of $\mathrm{Co}: \mathrm{Fe}=1: 2$.

4. CNP treatment has played an important role in regulating the morphology and magnetic properties of cobalt ferrite nanopowders.

5. $\mathrm{CoFe}_{2} \mathrm{O}_{4}$ nanopowders synthesized by CNP treatment have a higher saturation magnetization and a coercive force.

\section{References}

[1] K. Maaz, A. Mumtaz, S.K. Hasanain, A. Ceylan, J. Magn. Magn. Mater. 308, 289 (2007).

[2] S. Amiri, H. Shokrollahi, Mater. Sci. Eng. 33, 1 (2013).

[3] O. Pekinchak, L. Vasylechko, I. Lutsyuk, Y. Vakhula Y. Prots, W. Carrillo-Cabrera, Nanoscale Res. Lett. 11, 75 (2016).

[4] L.A. Frolova, A.A. Pivovarov, High Energ. Chem. 49, 10 (2015).

[5] M. Sangmanee, S. Maensiri, Appl. Phys. A 97, 167 (2009).

[6] L. Frolova, A. Pivovarov, E. Tsepich, Nanophys. Nanophoton. Surf. Stud. Appl. 183, 213 (2016).

[7] L. Frolova, A. Derimova, I. Galivets, M. Savchenko, A. Khlopytskyi, EEJET 84, 64 (2016).

[8] D. Biswal, B.N. Peeples, C. Peeples, A.K. Pradhan, J. Magn. Magn. Mater. 345, 1 (2013).

[9] J. Philip, T. Jaykumar, P.K. Sundaram, B. Raj, Meas. Sci. Technol. 14, 1289 (2003).

[10] T. Dippong, E.A. Levei, G. Borodi, F. Goga, L.B. Tudoran, J. Therm. Anal. Calorim. 119, 1001 (2015).

[11] X.H. Li, C.L. Xu, X.H. Han, L. Qiao, T. Wang, F.S. Li, Nanoscale Res. Lett. 5, 1039 (2010).

[12] I. Sharifi, H. Shokrollahi, M.M. Doroodmand, R. Safi, J. Magn. Magn. Mater. 324, 1854 (2012). 